\title{
Petechial Rash on the Thighs in an Immunosuppressed Patient
}

Angie Y. Wan, BA; Melissa Piliang, MD

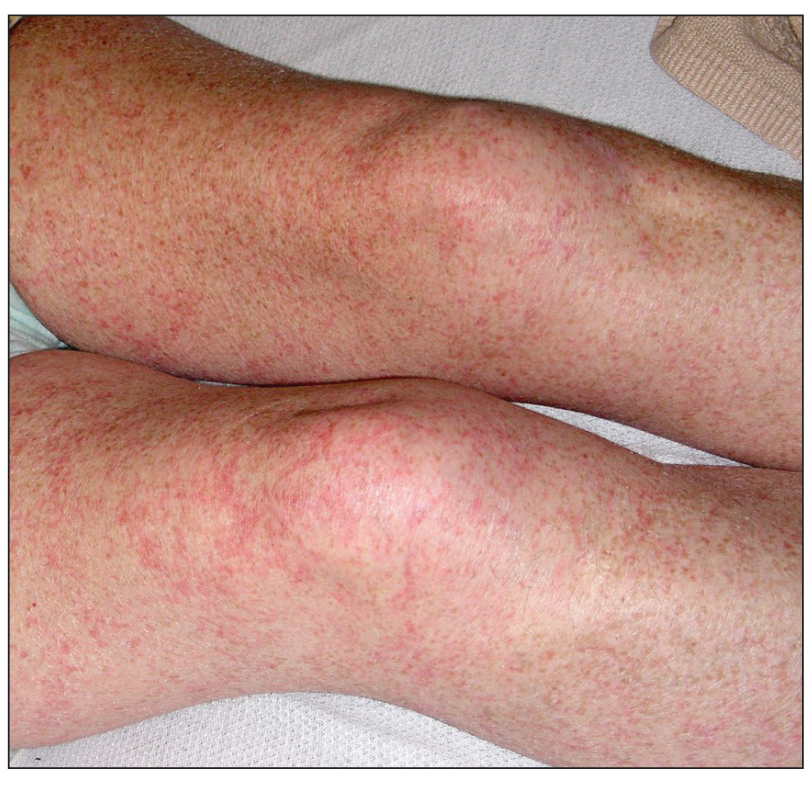

A 48-year-old woman from rural Virginia presented with centrifugally spreading, pruritic, blanchable macules over the lower abdomen and upper thighs noted 4 months after a pancreas transplant. After 3 weeks, the macules coalesced into reticulated nonblanching petechial patches. Fever, dyspnea, increasing xerosis, abdominal pain, and constipation were present. The patient had a medical history of type 1 diabetes mellitus requiring a pancreas transplant. Initial skin biopsy and fluorescence in situ hybridization to test for immune reaction to the XY-donor pancreas were negative. Mild transient eosinophilia was present at admission.

\section{WHAT'S YOUR DIAGNOSIS?}
a. disseminated intravascular coagulation
b. disseminated strongyloidiasis
c. drug-induced urticaria
d. graft-vs-host disease
e. viral exanthema

\footnotetext{
From the Department of Dermatology and Dermatopathology, Cleveland Clinic Foundation, Ohio. Ms. Wan also is from Case Western Reserve University School of Medicine, Cleveland.

The authors report no conflict of interest.

Correspondence: Angie Y. Wan, BA, Cleveland Clinic Foundation, Department of Dermatology and Dermatopathology, A60, 9500 Euclid Ave, Cleveland, OH 44195 (ayw16@case.edu).
} 


\section{THE DIAGNOSIS:}

\section{Disseminated Strongyloidiasis}

S trongyloidiasis is a parasitic infection caused by Strongyloides stercoralis. In the United States it is most prevalent in the Appalachian region. During the filariform larval stage of the parasite's life cycle, larvae from contaminated soil infect the human skin and spread to the intestinal epithelium, ${ }^{1}$ then the larvae mature into adult female worms that can produce eggs asexually. Rhabditiform larvae hatch from the eggs and are either excreted in the stool or develop into infectious filariform larvae. The latter can cause autoinfection of the intestinal mucosa or nearby skin; in addition, if the larvae enter the bloodstream, they can spread throughout the body and lead to disseminated strongyloidiasis and hyperinfection syndrome. ${ }^{2}$ This often fatal progression most commonly occurs in immunosuppressed individuals. ${ }^{3}$ The mortality rate has been reported to be up to $87 \% .{ }^{2,4}$

Fever, abdominal pain, nausea, and diarrhea are clinically common in disseminated strongyloidiasis and hyperinfection syndrome. ${ }^{5}$ Patients also may exhibit dyspnea, cough, wheezing, and hemoptysis. ${ }^{2}$ Cutaneous manifestations are rare and typically include pruritus and petechiae. ${ }^{6}$ Eosinophilia may be present but is not a reliable indicator. ${ }^{1}$

Our patient displayed several risk factors and an early clinical presentation for disseminated strongyloidiasis and hyperinfection syndrome, which evolved over the course of hospitalization. Clues to the diagnosis included an immunosuppressed state; erythematous pruritic macules at presentation that later developed into reticulated petechial patches; and fever, general abdominal symptoms, and dyspnea. However, the patient's overall physical examination findings were subtle and nonspecific. Additionally, the patient did not display the classic larva currens for strongyloidiasis or the pathognomonic periumbilical thumbprint purpura of disseminated infection, ${ }^{6,7}$ which may indicate that the latter is a later-stage finding. Although graft-vs-host disease initially was suspected, a third skin biopsy revealed basophilic Strongyloides larvae, extravasated erythrocytes, and mild perivascular inflammation (Figure).

Subsequent gastric aspirates and stool cultures revealed $S$ stercoralis. A bronchoalveolar lavage specimen and serum enzyme-linked immunosorbent assay for Strongyloides antibody were negative. The patient was treated with an extended 16-day course of ivermectin $12 \mathrm{mg}$ daily until gastric aspirates and stool cultures were negative for the parasite. The rash receded by the end of the patient's 32-day hospital stay.

Because of the high mortality rate of untreated disseminated strongyloidiasis and hyperinfection syndrome,
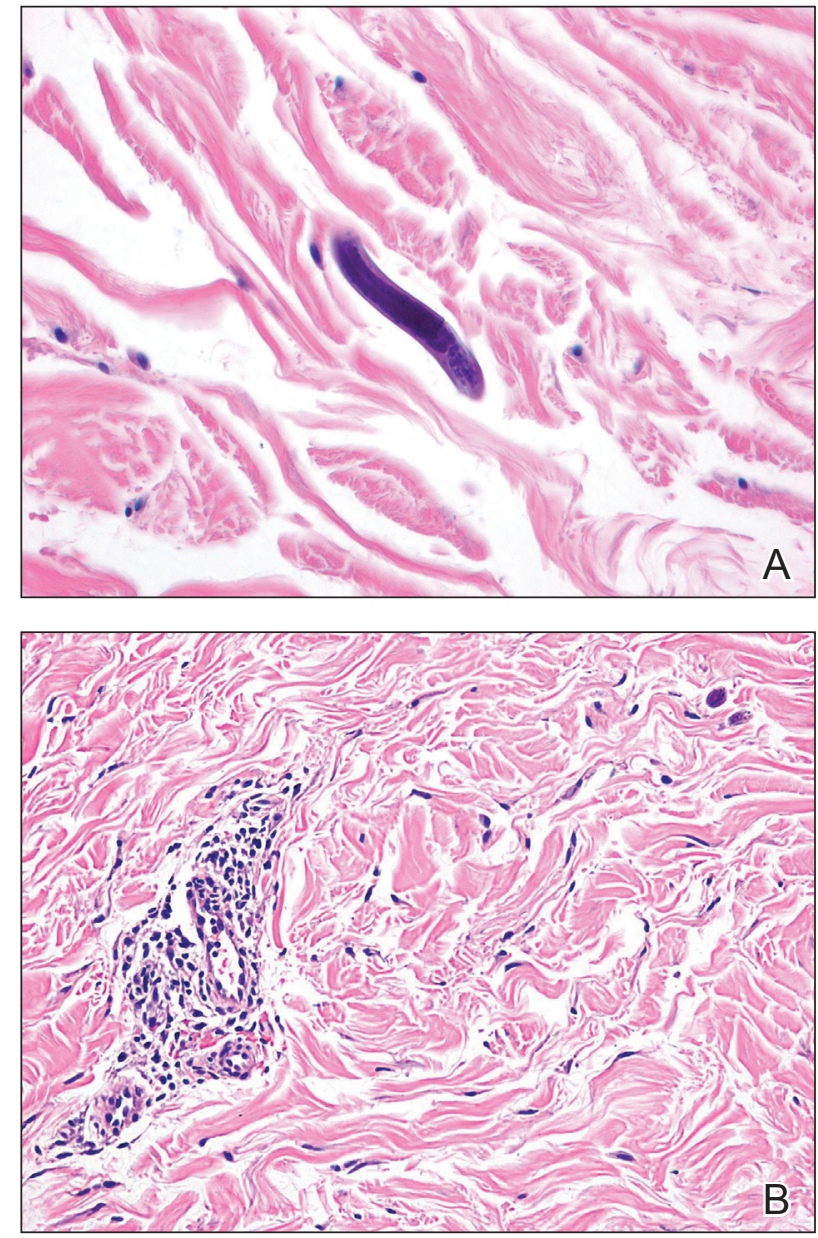

A, Skin biopsy showed Strongyloides larvae percolating between collagen bundles in the dermis (H\&E, original magnification $\times 40$ ). $B$, Perivascular inflammation and extravasated red cells were noted as well as Strongyloides larvae (H\&E, original magnification $\times 20$ ).

early diagnosis and initiation of anthelmintic treatment is vital in improving patient outcomes. As such, the diagnosis of disseminated strongyloidiasis should be considered in any immunosuppressed patient with multisystemic symptoms and/or petechiae. The differential diagnosis includes graft-vs-host disease, drug-induced urticaria, disseminated intravascular coagulation, and other opportunistic parasites. ${ }^{6,8,9}$

\section{REFERENCES}

1. Concha R, Harrington W Jr, Rogers AI. Intestinal strongyloidiasis: recognition, management, and determinants of outcome. J Clin Gastroenterol. 2005;39:203-211.

2. Vadlamudi RS, Chi DS, Krishnaswamy G. Intestinal strongyloidiasis and hyperinfection syndrome. Clin Mol Allergy. 2006;4:8. 
3. Keiser PB, Nutman TB. Strongyloides stercoralis in the immunocompromised population. Clin Microbiol Rev. 2004;17:208-217.

4. Chan FLY, Kennedy B, Nelson R. Fatal Strongyloides hyperinfection syndrome in an immunocompetent adult with review of the literature. Intern Med J. 2018;48:872-875.

5. Scowden EB, Schaffner W, Stone WJ. Overwhelming strongyloidiasis: an unappreciated opportunistic infection. Medicine (Baltimore). 1978;57:527-544.

6. von Kuster LC, Genta RM. Cutaneous manifestations of strongyloidiasis. Arch Dermatol. 1988;124:1826-1830.
7. Weiser JA, Scully BE, Bulman WA, et al. Periumbilical parasitic thumbprint purpura: Strongyloides hyperinfection syndrome acquired from a cadaveric renal transplant. Transpl Infect Dis. 2011;13:58-62.

8. Berenson CS, Dobuler KJ, Bia FJ. Fever, petechiae, and pulmonary infiltrates in an immunocompromised Peruvian man. Yale J Biol Med. 1987;60:437-445.

9. Ly MN, Bethel SL, Usmani AS, et al. Cutaneous Strongyloides stercoralis infection: an unusual presentation. J Am Acad Dermatol. 2003;49(2 suppl case reports):S157-S160. 